\title{
An experimental study into the effect of transitional flow on the performance of underwater glider wings
}

\author{
Artur K. Lidtke*, Simon Lewis ${ }^{\dagger}$, Terry J. Harvey ${ }^{\ddagger}$, Stephen R. Turnock* and Jon Downes* \\ ${ }^{*}$ Fluid-Structure Interactions Group, University of Southampton, Southampton, SO16 7QF, UK \\ ${ }^{\dagger}$ CJR Propulsion Ltd. 70-72 Quayside Rd, Southampton, SO18 1AD, UK \\ $\ddagger$ Engineering and the Environment, University of Southampton, Southampton, SO17 1BJ, UK
}

\begin{abstract}
The experimental procedures developed to measure small forces generated by underwater vehicle appendages in a wind tunnel are described. These involve using precision load cell sensors arranged into a bespoke dynamometer, that has been integrated into a stand-alone wind tunnel rig. The set up is used to quantify performance of a scaled underwater glider hydrofoil, with particular care being paid to the effects of laminar and transitional flow features. Present data are benchmarked against similar measurements and simulations and it is shown that satisfactory results may be obtained with the current approach despite its simplicity and low cost. The apparatus is then used to show that the presence of laminar flow significantly affects performance of the considered foil at Reynolds numbers corresponding to a typical regime in which underwater gliders operate.
\end{abstract}

\section{INTRODUCTION}

Underwater gliders are vehicles travelling through the water by converting their vertical motion induced via controlling their net buoyancy into a useful thrust force. In order to achieve this they rely on hydrofoils whose efficiency dictates how big a buoyancy engine the vehicle must be fitted with in order to achieve the desired forward speed and glide path angle. This directly affects the amount of energy expended on propulsion by means of operating the buoyancy engine pumps.

Most common underwater gliders travel at speeds typically below $0.5 \mathrm{~ms}^{-1}$ as their primary missions prioritise endurance over maximum speed. This implies that Reynolds numbers experienced by their appendages are expected to be in the range of 100,000 and less [1], [2], and are thereby likely to be affected by laminar and transitional flow phenomena, which will have a major effect on the performance of the vehicle propulsion.

Accurately accounting for these effects has been shown to be vital to provide a realistic estimate of expected vehicle performance using computational fluid dynamics [3]. A related study by Lemaire et al. [4] has predicted a significant amount of complex, three-dimensional fluid features to exist on a representative underwater glider wing. The numerical results indicated that both the low Reynolds number and moderate aspect ratio play a significant role in effecting the incipient flow patterns. This has also been confirmed by published experimental studies. For instance, several authors investigated the effect of low aspect ratios at low Reynolds numbers. Among those, studies which stand out are those by Parras \& Pino [5], Ananda et al. [6], Bastedo \& Mueller [7], and Mizoguchi \& Hajime [8]. All of the authors studied different foils and planforms, but have generally concluded that trailing edge vortices interact significantly with laminar and transitional flow features, which in turn affects the overall perofmance of the wings. It is a known fact that the tip vortex affects the local angle of attack of the foil. Bastedo \& Mueller stipulate that this modifies the shape and extend of the laminar separation bubble as this is said to behave in a two-dimensional fashion in accordance with the local inflow angle. A significant amount of research effort has been put into studying low-Re behaviour of flows past insect-like wings [8], [9]. These also report very high dependence of aerodynamic performance on the planform shape. Far fewer studies investigated the effect of sweep angle, which is also characteristic of underwater glider geometries. One of those is the study by Yen \& Huang [10] considered swept wings based on a NACA 0012 profile using oil flow visualisations and found the sweep angle to significantly affect the nature and size of laminar separation bubbles.

Therefore, the present work aims to deliver a cost-effective and targeted set of experiments to fill this gap and investigate performance of low aspect ratio, swept wings operating at low Reynolds numbers. The main challenge stems from the difficulty in measuring small forces generated by foils in the low-speed regime, which applies particularly to drag. Present work therefore describes the techniques used in order to tackle this issue. Furthermore, the process of manufacturing a hydrofoil intended to be used on a novel underwater glider design is presented. This employs $3 \mathrm{D}$ printing in order to minimise the experimental campaign costs by abandoning traditional, labour-intensive model-making techniques. Accuracy of the models is quantitatively assessed using laser scanning. A low-speed wind tunnel of the University of Southampton is then used in order to provide force measurements. The obtained results are benchmarked against available data for two-dimensional low-Re foils in order to judge the ability of the present experimental rig to measure the drag force directly. In the future, due to the relatively low cost of manufacturing new models, the test campaign will also involve optimising the hydrofoils and rudders for the new vehicle being designed as part of the EU BRIDGES Project (http://www.bridgesh2020.eu/). 


\section{EXPERIMENTAL SET UP}

\section{A. Force acquisition system design}

The key objective of designing the experimental set up was to ensure it is sensitive enough in order to capture the drag force, which was expected to be an order of magnitude less than the side force. In their experiments on a series of 2D low-Re aerofoils, Selig et al. [11] used load cells to measure lift but relied on calculating the drag through momentum analysis of the foil wake, precisely because of the difficulty in measuring the small forces directly. Such an approach is potentially more accurate, but is difficult to apply to three-dimensional geometries, may be less precise for stall performance estimates, and requires a more complex experimental set up.

Therefore, miniature transducers typically used in precision scales were selected to be used in the force acquisition system. These have a rated load of $1 \mathrm{~kg}$, rated output of $1 \mathrm{mV} / \mathrm{V}$ and are powered from a $5 \mathrm{~V} \mathrm{DC}$ power supply. Outputs are amplified using HX711 load cell amplifiers, and the signals are collected using analog pins on an Arduino Uno micro controller operating at a rate of $500 \mathrm{~Hz}$. The information is then passed on to Python-based acquisition software running on a laptop via the serial port.

The angle of attack was adjusted automatically using a $5 \mathrm{~V}$ stepper motor. This offers a resolution of only 200 steps per revolution, but this is improved by employing a 5.8:1 gear ratio, which translates to incidence angle adjustment resolution of approximately $0.324^{\circ}$. A key factor in the rig assembly was to minimise the backlash in the gears to minimise vibration on the foil and limit its unintentional pitching motion. An independent angle of attack measurement is achieved by using a Broadcom rotary encoder (HEDS-9040) and an encoder wheel with 1000 pulse resolution (HEDS-6140). These are used to verify the requested angle of the foil.

In total, six transducers are used - two for measuring the axial and four for acquiring the side force. They form a force block which rotates with the foil which simplifies the rig design and allows the use of smaller, more precise but more frail sensors. A schematic of the employed arrangement is depicted in Figure 1. The final force block is also shown in Figure 2. Figure 3 shows the final arrangement of the rig mounted underneath the wind tunnel working section. Neither the foil nor any other elements of the acquisition system are in contact with the wind tunnel structure, which reduces vibration and limits spurious readings on the sensitive load cells. This is achieved by the foil being mounted on two aluminium rods penetrating small cut-outs in the wind tunnel floor which allow for the angle of attack to be adjusted but do not lead to a significant leakage of fluid momentum. Although this functionality is not yet implemented, the hardware and software design should allow for the tests to be fully automated, which would reduce the costs of the testing campaign, reduce human errors, and improve the utilisation efficiency of the wind tunnel facility.

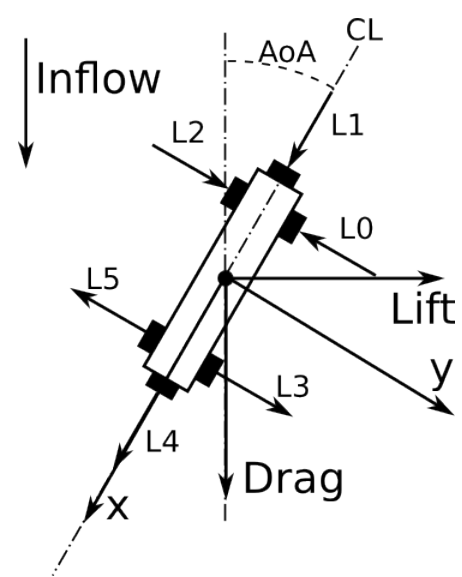

Fig. 1. Diagram of the force transducer arrangement and positive lift, drag, and angle of attack directions. Arrows illustrating load on each of the six load cells indicate its individual positive measurement direction.

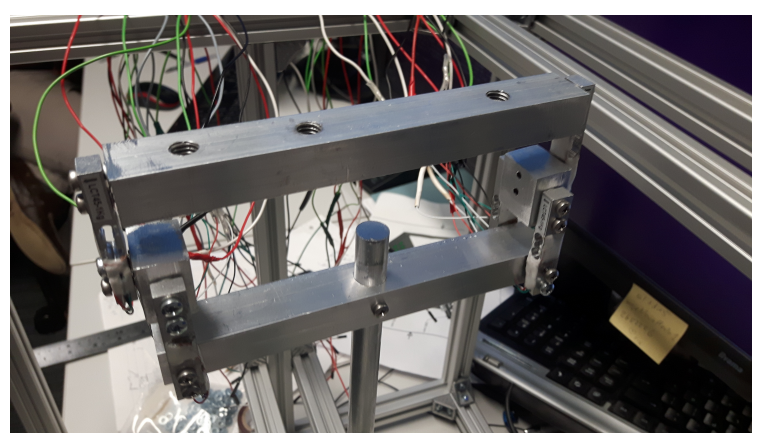

Fig. 2. Photograph showing the force block during assembly of the test rig.

The transducers measure loads along a single axis each by relating the strains of the aluminium beams to the force applied. This implies that a total force is computed by summing the readings along each principle axis in the foil reference system. By comparing the readings of side force transducers upstream and downstream of the axis of rotation pitching moment may be deduced, although this is not considered in the current work. Once the total force in the force block reference frame has been obtained, it has to be resolved onto the drag and lift axes fixed with respect to the wind tunnel,

$$
\begin{aligned}
& L=-F_{X} \sin (\alpha)+F_{Y} \cos (\alpha), \\
& D=F_{X} \cos (\alpha)+F_{Y} \sin (\alpha),
\end{aligned}
$$

where $\alpha$ is the angle of attack measured from the wind tunnel axis, $L$ and $D$ are the lift and drag, while $F_{X}$ and $F_{Y}$ refer to the axial and side forces in the foil reference frame.

\section{B. Calibration}

A key step in developing the acquisition hardware involved calibrating the response of the force block when subject to known lift and drag forces. These were exerted through hanging of precision weights via a set of pulleys, following a similar approach to the one discussed by Molland [12]. This 


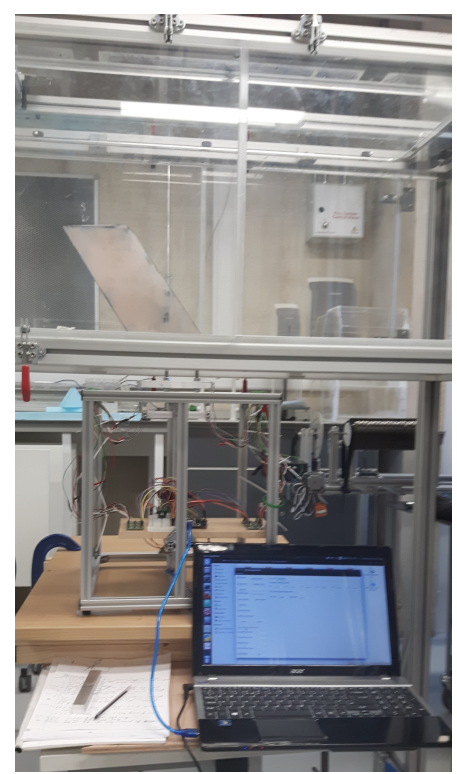

Fig. 3. Finished rig fitted with a foil placed underneath the wind tunnel working section.

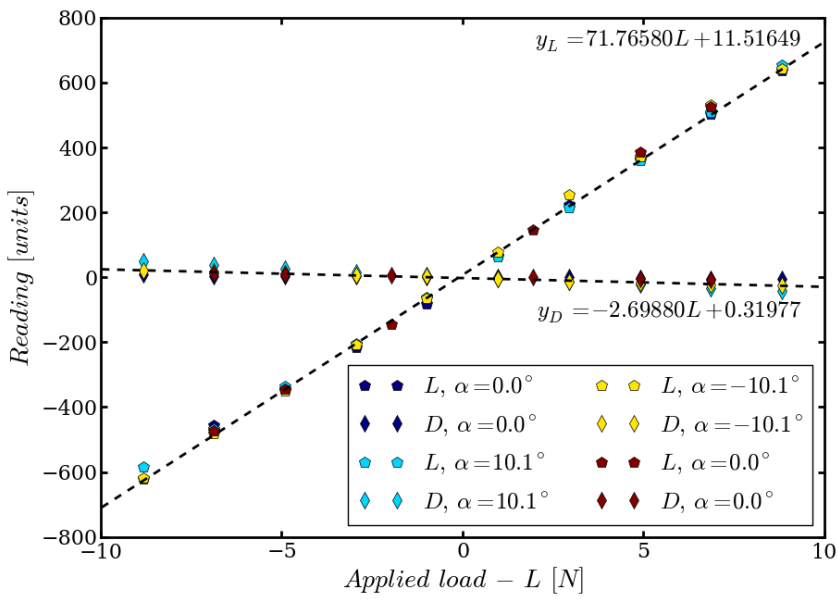

Fig. 4. Response of the force block to known loads applied in the lift direction, also showing the induced false drag readings caused by load cell interactions.

process was repeated at three different angles of attack: -10 , 0 and $10^{\circ}$, which correspond to the regime where the foil is not expected to stall. The forces were applied in isolation, meaning only pure lift (positive and negative) and pure drag were considered. Mass of the weights had been verified down to 1 gram, which is of magnitude comparable to the forces measured on the foils at lowest Reynolds numbers. Figure 4 shows the rig response to applied lift loads. An analogous result was obtained for drag which is not presented for brevity.

It has been observed that the angle of attack has little effect on the measured forces. Furthermore, it is expected that at zero load there will be no force being measured, and hence the linear fits to the data should intercept the y-axis at 0 . This is not the case in practice due to errors in the measurement of drag, misalignments of the calibration set up, as well as uncertainties of the measurements. However, the obtained values are small enough to be acceptable at this stage. Importantly, two series of data at zero angle of attack, obtained during two separate calibration experiments, show very little variation, indicating good repeatability of the measurements, at least in a controlled environment.

From the slopes of the response curves one may deduce the uncorrected lift and drag values,

$$
\begin{aligned}
L_{u} & =\left(\frac{\mathrm{d} y_{L}}{\mathrm{~d} L}\right)^{-1}=0.013934 \frac{N}{\text { unit }}, \\
D_{u} & =\left(\frac{\mathrm{d} y_{D}}{\mathrm{~d} D}\right)^{-1}=0.013408 \frac{N}{\text { unit }} .
\end{aligned}
$$

Interference in the readings, in the form of a lift signal being measured when pure lift is applied and vice versa, also varies linearly with the applied load, namely

$$
\begin{aligned}
& A_{D L}=\frac{\mathrm{d} y_{L}}{\mathrm{~d} D} / \frac{\mathrm{d} y_{L}}{\mathrm{~d} L}=0.024492 \frac{N}{N o f d r a g}, \\
& A_{L D}=\frac{\mathrm{d} y_{D}}{\mathrm{~d} L} / \frac{\mathrm{d} y_{D}}{\mathrm{~d} D}=-0.036185 \frac{N}{N \text { of lift }} .
\end{aligned}
$$

This means that pure lift generates a reading of approximately $3.9 \%$ of the applied value in the drag axis. The drag-tolift coupling is at the level of approximately $2.5 \%$ of the applied value. These values may appear small, but one has to appreciate that expected magnitudes of the drag forces are far less than those of lift. Therefore, in a real experiment the drag reading due to lift may form a significant proportion of the registered signal, particularly at higher angles of attack. This is illustrated in Figure 5, which presents raw axial and side force readings recorded during one of the measurements that will be described in the following parts of this paper. Notably, the side force curve resembles the typical shape of a lift curve, but the measured axial values do not, precisely due to the interaction effects caused by lift.

Making use of Equations (2) and (3), the lift and drag values may corrected for the interaction effects as

$$
\begin{aligned}
& L_{c}=L_{u}-A_{D L} D_{c}, \\
& D_{c}=D_{u}-A_{L D} L_{c} .
\end{aligned}
$$

This expression is not particularly useful as it contains both corrected and uncorrected values on the right hand side, while the aim of the calibration process is to arrive at the corrected values given the raw, uncorrected readings. Therefore, the equations are written in a matrix format and rearranged,

$$
\left[\begin{array}{cc}
1 & A_{D L} \\
A_{L D} & 1
\end{array}\right]\left[\begin{array}{c}
L_{c} \\
D_{c}
\end{array}\right]=\left[\begin{array}{c}
L_{u} \\
D_{u}
\end{array}\right] .
$$

The coefficient matrix on the left-hand side may now be inverted and the equation solved to yield corrected values based on uncorrected readings. For the present experimental set up, this yields

$$
\left[\begin{array}{l}
L_{c} \\
D_{c}
\end{array}\right]=\left[\begin{array}{rr}
0.99942 & -0.01567 \\
0.03687 & 0.99942
\end{array}\right]\left[\begin{array}{l}
L_{u} \\
D_{u}
\end{array}\right] .
$$




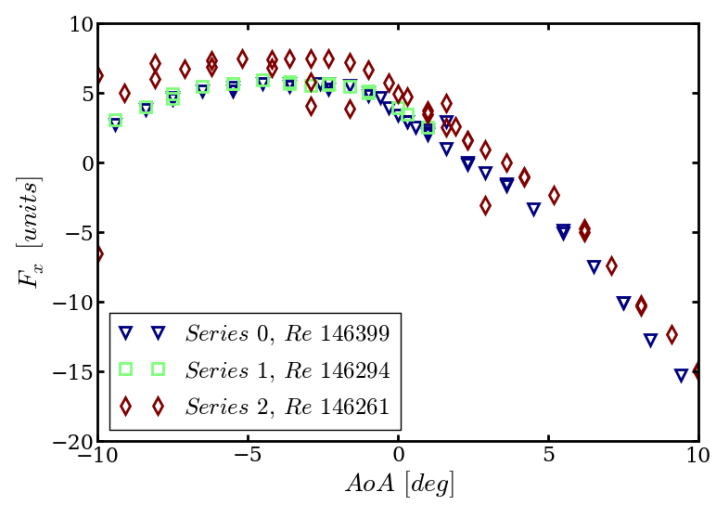

(a) Axial force

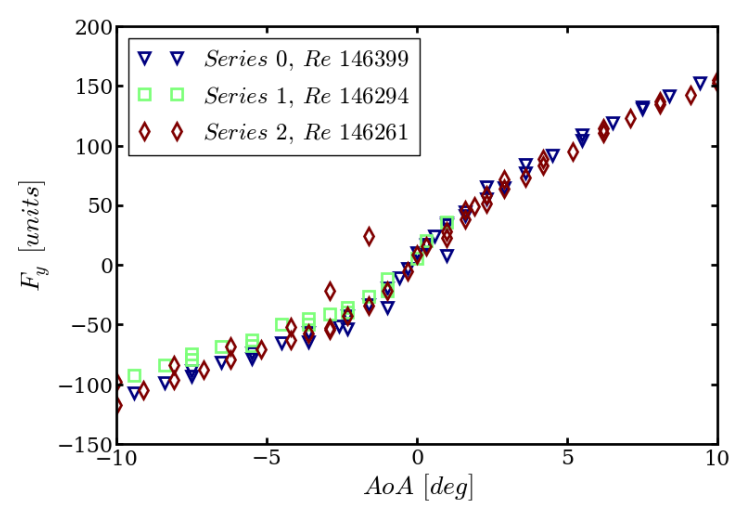

(b) Side force

Fig. 5. Raw readings as registered by the transducers before they are resolved into the tunnel axis system and interactions effects between the two principle directions are corrected for. Data shown for three different acquisition runs.

\section{Sources of error and uncertainty}

Several possible sources of error have been identified in the present experimental procedure. Firstly, the angle of attack of the foil was determined based on the no-lift condition rather than measured with respect to the wind tunnel axis. While this would have been acceptable for a perfectly symmetrical foil, the present geometry has been shown not to be such. Furthermore, vertical alignment of the foil was shown to affect its performance significantly. While care has been taken to ensure proper alignment, this was done manually using a spirit level and hence some misalignments could have persisted. The angle of attack adjustment and measurement accuracy are also lower than would have been desirable.

Furthermore, the force acquisition system itself is not uncertainty-free. The force block had been manufactured and assembled with care, but due to the manual nature of this task lack of symmetry and misalignments in the mounting of the sensors were difficult to avoid completely. These could potentially lead to non-linear behaviour, especially for the interaction terms, which may not be easily factored into the data handling procedures. Despite their small size and rated load, the transducers used had difficulties with capturing the very small forces present at lower of the considered Reynolds numbers, leading to poor signal-to-noise ratios. Importantly, due to their small magnitude, the drag readings were significantly affected by the interaction terms due to lift. Although these had been quantified and accounted for, inaccuracies in the calibration process could have affected the end results to a considerable extent.

\section{TEST METHOdOLOGY}

\section{A. Geometry and test conditions}

The considered foil is a scaled-down version of a wing representative of what is intended to be fitted to a new underwater glider design. This is identical to the one previously studied numerically by Lemaire et al. [4] and employs a modified SD8020 symmetric low-Re wing section. The modifications predominantly involved accommodating a trailing edge of finite thickness suitable for manufacturing. Planform of the foil is summarised in Figure 6.

Size of the wing has been chosen in order to allow the Reynolds numbers expected on the full-scale glider to be achieved in the available test facility. These were approximately 60,000, 100,000, 120,000, and 146,000, and cover the full operational range of the vehicle.

The foil was manufactured using a BigRep ONE 3D printer in PLA plastic. Deposition height of $0.7 \mathrm{~mm}$ was used, which led to a relatively rough surface finish. The surface of the foil was therefore later sanded and smoothed through application of a filler resin.

\section{B. Assessing manufacturing accuracy}

A key aspect of the model making process was assessing how accurately the finished foil represents the design intent. Main concerns stemmed from the fact that 3D printed objects are known to undergo thermal distortion due to different cooling rates throughout the body, but also from the manual finishing of the model.

Laser scans were therefore carried out using HandyScan 300 device with linear accuracy of $0.04 \mathrm{~mm}$ and volumetric accuracy of $0.0 \mathrm{~mm}$. This was used to produce a stereolitography (stl) representation of the final foil. This was aligned with the original geometry in order to minimise the errors between key points on the foil - mounting holes, trailing edge and tip features. B-spline curves were then fitted to the scans using proprietary surface provided by the laser scan manufacturer. These could then be compared with cuts through the original intended model, as well as the ideal foil offsets (excluding the finite trailing edge), as shown in Figure 7.

It may be seen that the leading edge profiles of the sections through the manufactured model agree well with the desired shape. However, from around the maximum thickness and further downstream the model differs from the intended shape. What is more important, is that it is visibly asymmetric about the centreline, which has the potential to affect its performance significantly by inducing effective camber on the foil. 


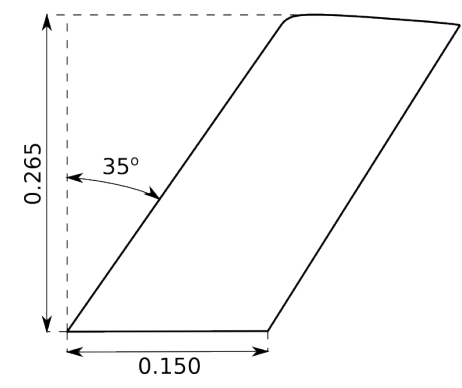

(a) Schematic - dimensions in metres

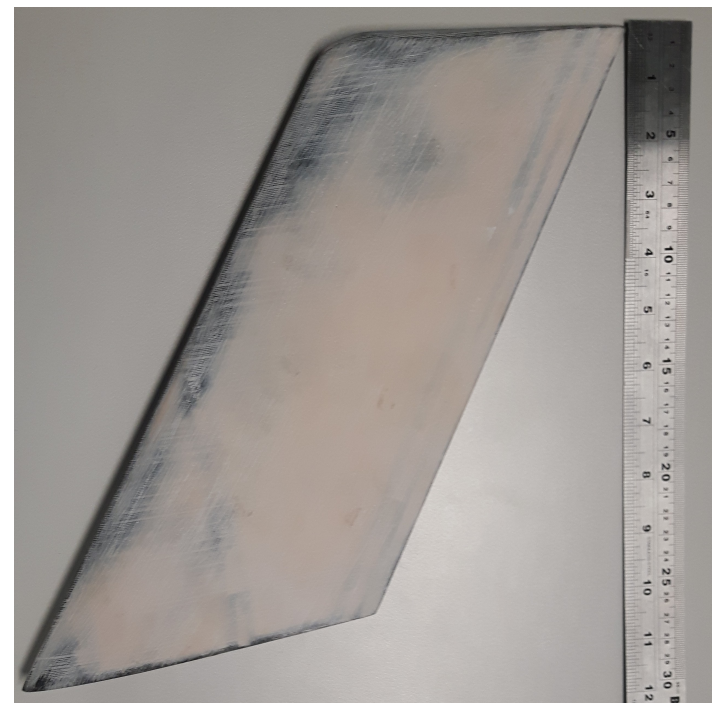

(b) Picture of the finished foil - rules scales in inches and centimetres

Fig. 6. Schematic drawing and photograph of the tested foil.

Another important characteristic of the model is its surface roughness. It will affect the rate of generation of turbulence and instabilities in the boundary layer, an thus affect the Reynolds number dependence of the aerodynamic response. In particular, flow transition from laminar to turbulent state will be influenced.

Surface finish was therefore investigated using TaylorHobson Form Talysurf-120L, a device which is capable of measuring the shape of a surface along a one-dimensional cut with very high precision. This is done by dragging a stylus along the surface and measuring its displacement, as depicted in Figure 8. The measurements were conducted at two locations - in the centre and around the tip of the foil. These had been identified by visual observation to be smooth and rough areas, respectively, in relation to the other parts of the foil. The results are depicted in Figure 9. It may be seen that the peaks on the surface had been successfully eliminated by sanding, although a large amount of roughness persists in the form of troughs. In the smoother part, the RMS value of surface profile to be around $3.7 \mu \mathrm{m}$. Around the tip and leading edge, where refining the finish by hand is more difficult, the foil is more rough with the RMS of surface profile of $7.8 \mu \mathrm{m}$.

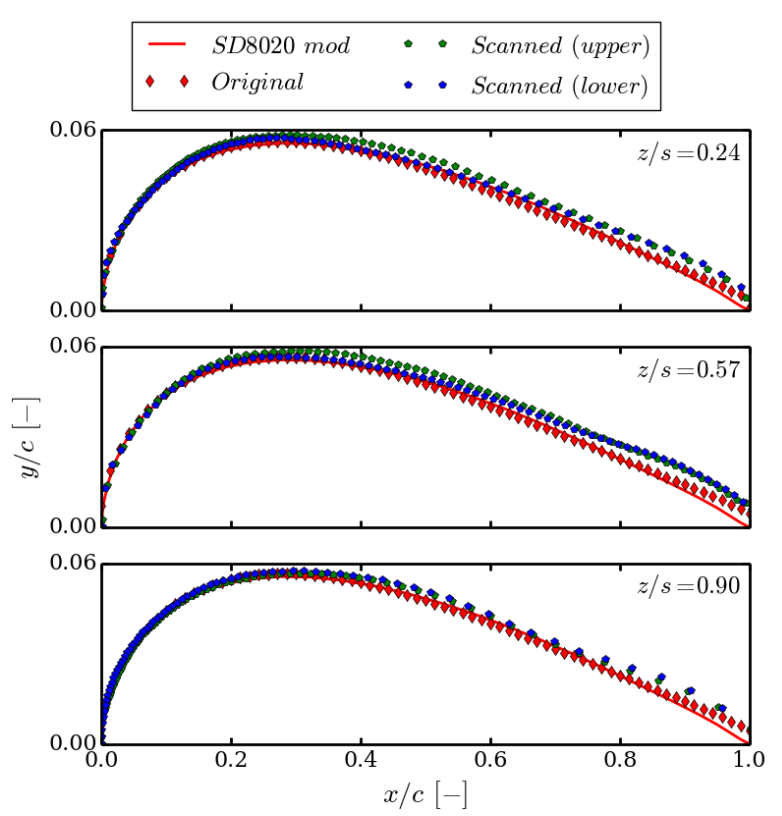

Fig. 7. Span-normal cuts through the foil, showing the idealised SD8020 shape ("SD8020 mod" series), the intended shape with a finite thickness trailing edge ("Original" series), as well as the real offsets laser-scanned off the test piece ("Scanned" series). Upper and lower surface of the latter shown separately to illustrate asymmetries caused by the manufacturing process.

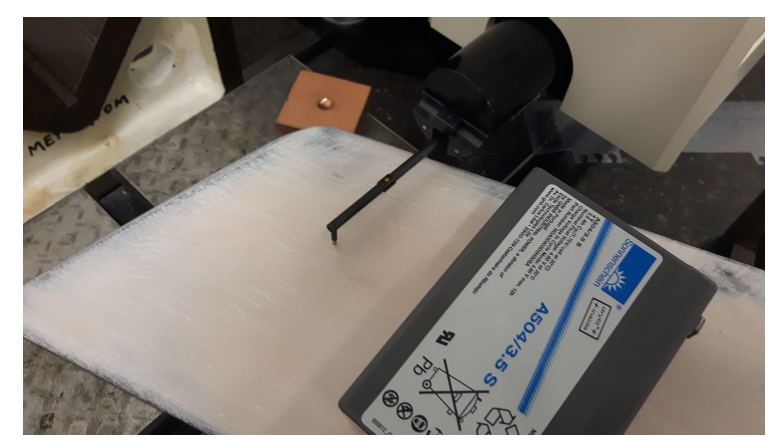

Fig. 8. Measuring the surface roughness of the foil - the black stylus is dragged across the surface of the foil and its displacement is recorded.

\section{The wind tunnel facility}

The wind tunnel used to conduct the tests has a working test section $450 \mathrm{~mm}$ high, $600 \mathrm{~mm}$ wide, and approximately $1000 \mathrm{~mm}$ long. The downstream end is open to the atmosphere, while the air is fed to the test section through a convergent nozzle fitted with turbulence reducing vanes. The foil is mounted on at the centreline of the bottom wall, approximately in the middle of the working section and $800 \mathrm{~mm}$ downstream of the convergent nozzle. Mean wind speed for each test run was measured using RS Pro 1340 hot-wire anemometer placed in the upstream part of the test section.

Each of the presented data points has been obtained by averaging data collected over an acquisition period of at least 30 seconds, or at least 1800 unit of non-dimensional time based on the working section length and flow speed. The 


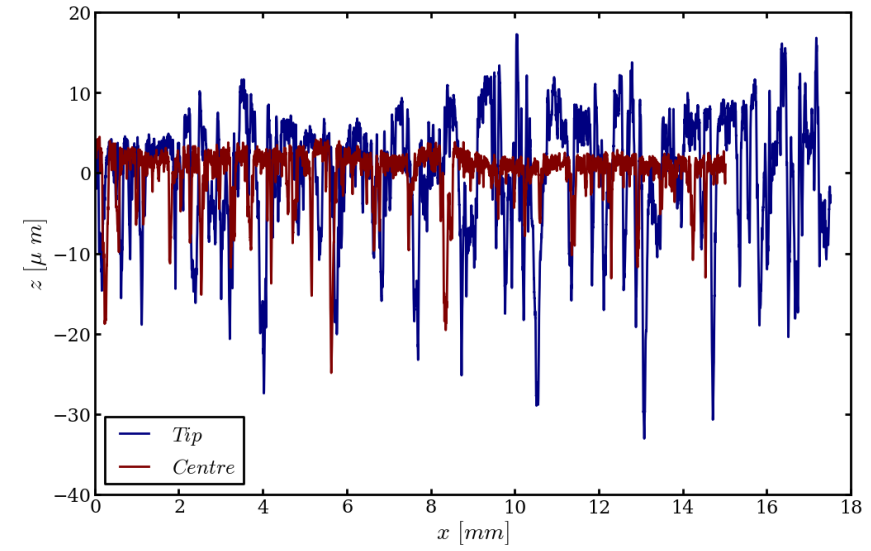

Fig. 9. Surface roughness on the surface of the foil at two locations - around the centre of the planform and closer to the tip, which had been visually identified as the least smooth area.

acquisition rate was $500 \mathrm{~Hz}$. The blockage ratio for the present model is under $2 \%$. Current data is presented without any blockage corrections as these will only be necessary once the results are to be scaled up to provide estimates of the foil in an unbounded flow.

Turbulence intensity in the working section of the wind tunnel used is in the range of $0.25-0.5 \%$ [13]. According to Selig et al. [11], a value of $0.1 \%$ is more appropriate to produce clearly defined laminar flow behaviour at low Reynolds numbers. Unfortunately, improving the existing facility was outside of the scope of work of this project. Nevertheless, it will be demonstrated that laminar flow behaviour was still observed on the considered wing.

\section{RESULTS}

\section{A. Effect of foil asymmetry}

The first part of the investigation focused on assessing the effect of the asymmetry and deviation from the intended shape of the foil, which had been identified through laser scanning the finished model. This was done by first calculating theoretical lift and drag coefficients for a 2D section of the foil using a Reynolds Averaged Navier Stokes (RANS) solver, following a similar approach to previous numerical work by the authors [3]. The k- $\omega$ SST turbulence model was used on structured O-grids with approximately 100,000 cells and wallresolved boundary layers. Predictions were made for the ideal shape with a sharp trailing edge, the desired geometry, and a scanned cut, as shown in Figure 10. Scanned cut $60 \mathrm{~mm}$ from the root of the foil was selected, as this was deemed to represent median deviation measured with the scanner.

As expected, increasing the trailing edge thickness does not affect the theoretical lift coefficient significantly, but has a pronounced effect on the increase in drag. As the net effect of the manufacturing inaccuracies appears to be an increased thickness in the aft part of the foil, they also effect higher drag of the real section. The conducted simulations do not account for asymmetry, but it may be seen that the deviations measured

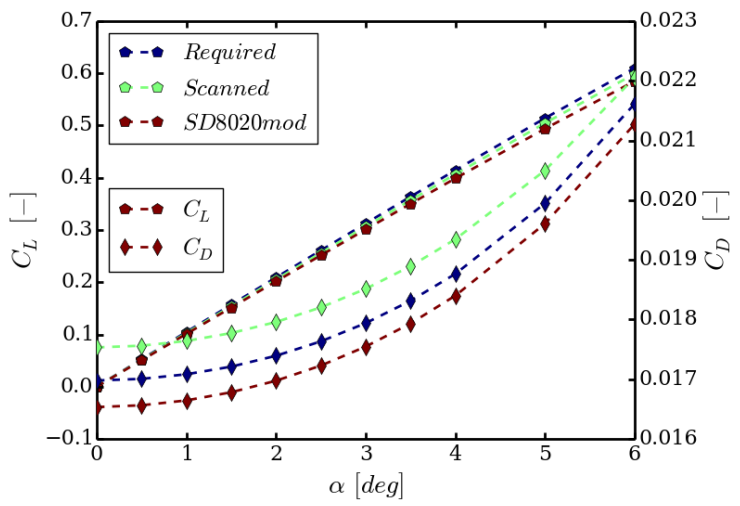

Fig. 10. Numerical prediction of the effect of foil shape on the performance of the foil. Data series correspond to the idealised SD8020, the intended finitewidth trailing edge geometry, and a profile scanned at $\mathrm{z} / \mathrm{s}$ of 0.24 . Simulations assumed the foil to be symmetric and did not account for laminar flow effects.

are significant enough to affect performance of a cut through the foil. It is hence likely that as the shape on the upper and lower sides of the $3 \mathrm{D}$ wing varies, these deviation may add up to noticeably different performance at positive and negative inflow angles.

Measurements in the wind tunnel were then conducted with the foil being subject to a range of angles of attack at the highest considered Reynolds number, so as to reduce the significance of low-Re flow features. The lift and drag data was then plotted in terms of absolute quantities, as shown in Figure 11. For a symmetric foil, data collected on both positive and negative angles of attack should collapse on top of each other, which may be seen not to be the case. This is a clear confirmation of the manufactured wing not being perfectly symmetric.

\section{B. Effect of alignment}

Potential for misalignment of the foil inside the wind tunnel had been identified as one of the major likely sources of error. Indeed, during one of the earlier tests highly asymmetric performance was measured, as seen in Figure 12. Upon closer investigation it was discovered that despite the test rig having been levelled prior to the tests, a relatively small inaccuracy in the alignment process had caused the foil to be heeled over. Once the alignment had been refined, a much more symmetric response had been recorded.

\section{Comparison with reference data}

A final test of the test set up involved validating the measured results against available benchmark data. For that purpose, the experimental results for a 2D SD8020 foil from the dataset by Selig et al. [11] was utilised. This could only be used in a qualitative manner, bearing in mind that the trailing edge of the present foil had been modified and that the manufactured wing differed in shape from the intended geometry. An attempt was also made to correct the 2D 


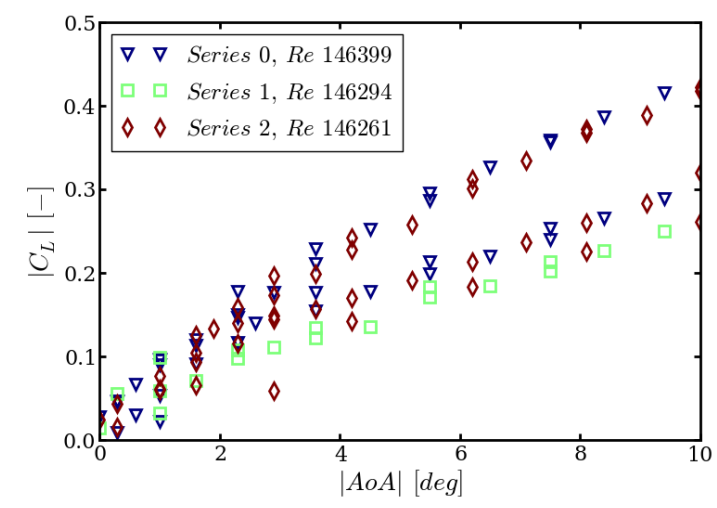

(a) Magnitude of lift coefficient and angle of attack

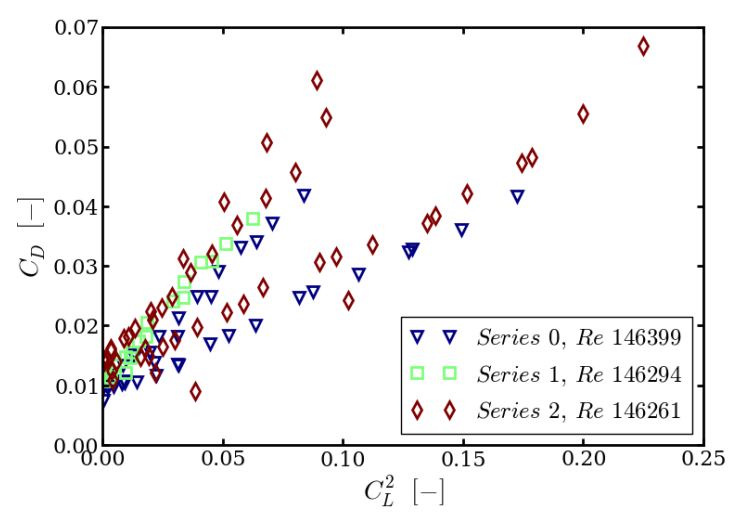

(b) Induced drag

Fig. 11. Absolute lift and drag values showing the effect of asymmetry of the foil. Data obtained with the wind tunnel operating at the lowest possible speed setting.

reference data to account for the finite aspect ratio of the present week, assuming that the three-dimensional lift and drag are

$$
\begin{aligned}
C_{D} & =C_{D 0}+k_{i} \frac{C_{L}^{2}}{\pi A R}, \\
C_{L} & =a_{i}\left[\alpha-\frac{57.3 C_{L}}{\pi A R}\right],
\end{aligned}
$$

where $k_{i}$ is the induced drag factor, assumed equal to 0.35 , $a_{i}$ is the lift curve slope, which was calculated using finite difference between $\pm 5^{\circ}$ angle of attack, $A R$ is the aspect ratio, and $C_{D 0}$ is the $2 \mathrm{D}$ drag coefficient [14]. Finally, results obtained by Lemaire et al. [4] for the present foil were also used as a benchmark. The comparison was done at the lowest considered Reynolds number in order to confirm the presence of laminar flow effects. The data are presented in Figures 13 and 14 for lift and drag coefficients, respectively.

Comparison of the presently measured lift coefficient to the two-dimensional SD8020 data by Selig et al. shows that indeed, laminar flow effects are present on the current foil, as evident from little to no lift being generated over approximately $4^{\circ}$. The extent of this behaviour qualitatively

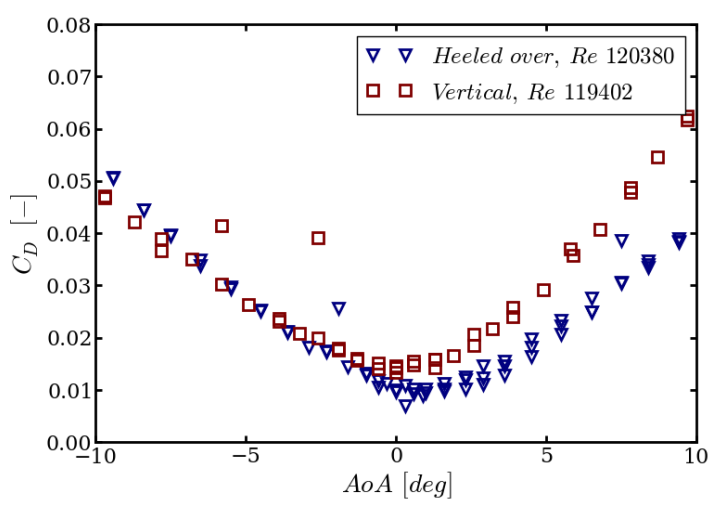

(a) Drag coefficient

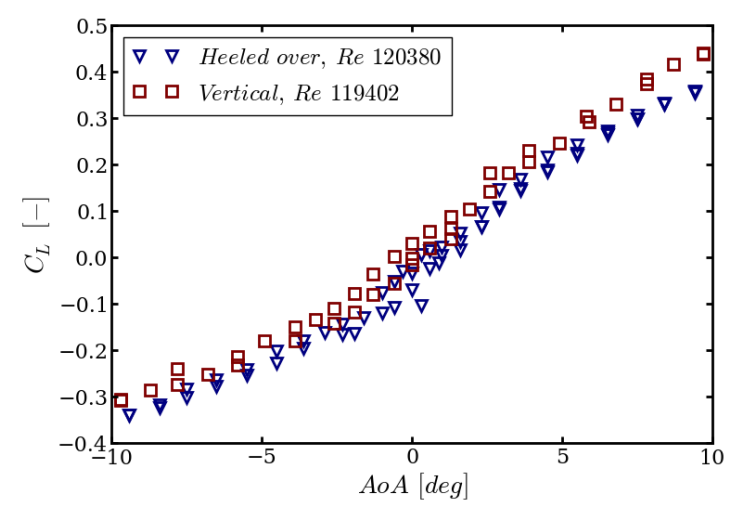

(b) Lift coefficient

Fig. 12. Effect of vertical foil alignment (heel) inside the tunnel on the predicted force coefficients.

matches the $2 \mathrm{D}$ foil data, although one may observe the flat part of the curve in the current data set to be shifted along the $\mathrm{x}$-axis, indicating asymmetric behaviour of the foil. Another important observation is the relatively good repeatability of the current data set when compared to the transducer drift reported for the reference data.

The process of correcting the $2 \mathrm{D}$ data to reflect the threedimensional foil performance is somewhat simplistic, and hence distorts the effect of laminar flow behaviour in the low angle of attack regime, as seen in Figure 13b. The slope of the curve beyond a few degrees does match the presently measured characteristics, as well as the numerical data from the literature. Noticeably, the currently recorded data may be seen to diverge from the corrected 2D curves more for the negative angles of attack, again hinting at asymmetric behaviour of the tested wing.

A crucial element of this part of the study was verifying the ability of the developed rig to measure drag coefficient accurately. This is explored in Figure 14, where recorded values are compared to corrected 2D data and computational results by Lemaire et al.. Much bigger qualitative discrepancies may be seen between the present and reference data, with the latter 


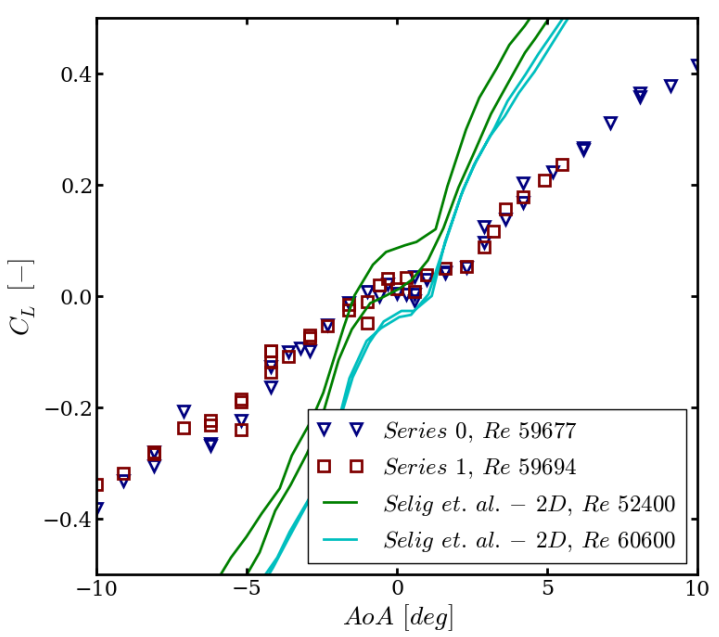

(a) Reference data for a 2D foil

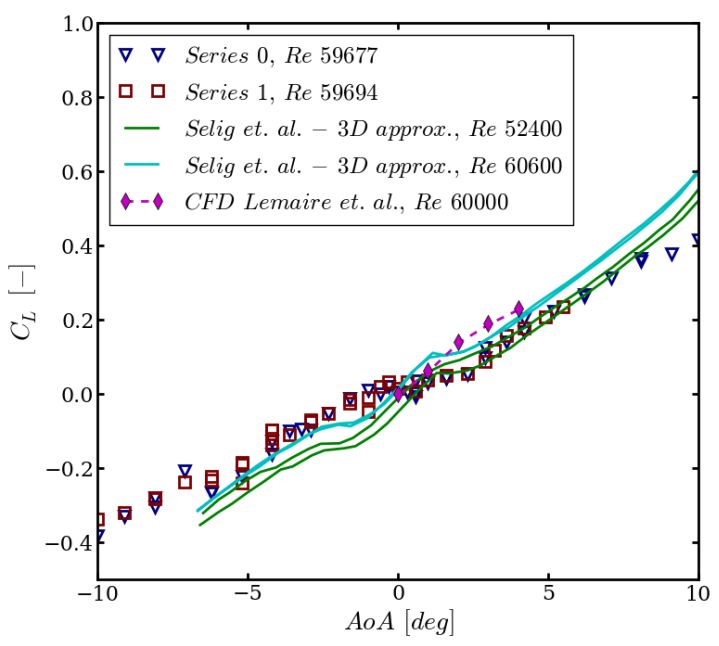

(b) Reference data corrected for a finite aspect ratio

Fig. 13. Comparison of the present data with experiments by Selig et al. [11] and numerical data by Lemaire et al. [4]. Reference experimental data presented both in raw form and corrected for a finite aspect ratio foil. Two lines in the reference measurements correspond to data collected for the foil being swept over a range of angles of attack in the positive and negative direction.

two agreeing relatively well. This indicates that at the lower Reynolds numbers considered in the current study the drag coefficient most likely may not be captured with sufficient accuracy. A notable observation is that nearly identical results were obtained for this quantity during two test runs conducted on two separate days. This may indicate that the transducers themselves are close to being sensitive enough, but the small magnitude of the forces being measured makes interaction effects and non-linearities in the force block response stand out, thus making the results less reliable.

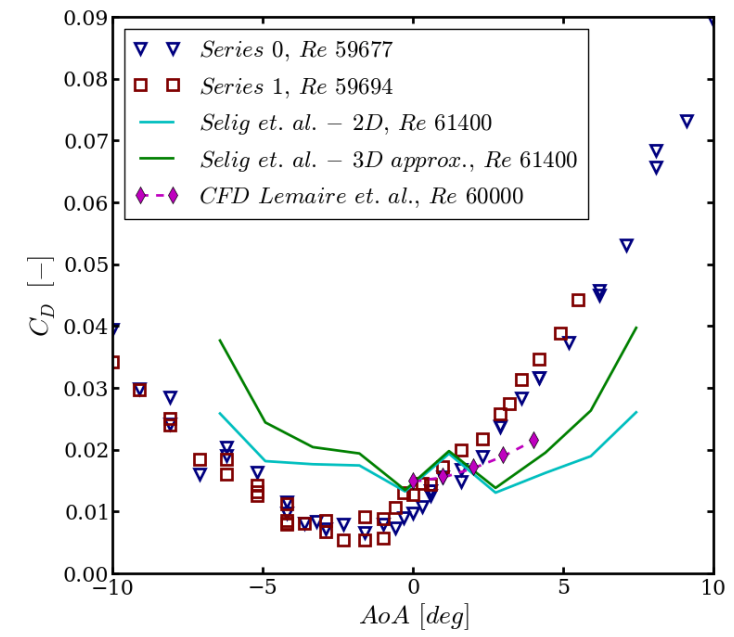

Fig. 14. Comparison with reference experimental data by Selig et al. [11] and numerical simulations by Lemaire et al. [4]. Reference experimental data corrected for a finite aspect ratio foil.

\section{Quantifying the Reynolds number effects}

The ultimate aim of the investigation was to study the effect of different Reynolds number regimes on the performance of the foil. It had been shown that the developed experimental set up should be capable of capturing the influence on the lift coefficient. While the accuracy of the drag measurements remains in question, they still provide a useful insight. Averaged results obtained for a number of series of data across four Reynolds numbers are presented in Figure 15.

The most important finding is the presence of a significant range of angles of attack for which the foil does not generate much lift at the lowest Reynolds number. As the inflow speed increases, the size of the dead zone in the lift coefficient curve reduces and disappears completely around Re of 120,000. Beyond this wind speed, the lift coefficient follows a straight line as a function of the angle of attack, as expected, but the slope of the curve is different on the positive and negative sides due to the asymmetry of the foil. This observed Reynolds number trend stands in very good qualitative agreement with the data presented by Selig et al. [11] for a two-dimensional SD8020 foil with a sharp trailing edge.

Measurements obtained for the drag coefficient indicate that at low Reynolds numbers the curve is asymmetric, with minimum drag coefficient occurring at approximately $-2^{\circ}$. As the Reynolds number goes up, the drag curves become more symmetrical. Beyond Re of 120,000 minimum drag coefficient occurs at $\alpha=0$ and the only asymmetries seen are in the shape of the curves away from origin. At lower Re the minimum drag coefficient is also lower than what had been computed by Lemaire et al. [4] and measured by Selig et al. [11] for a 2D foil. This does not appear realistic, as it had already been shown that on average the modifications, intended and not, to 


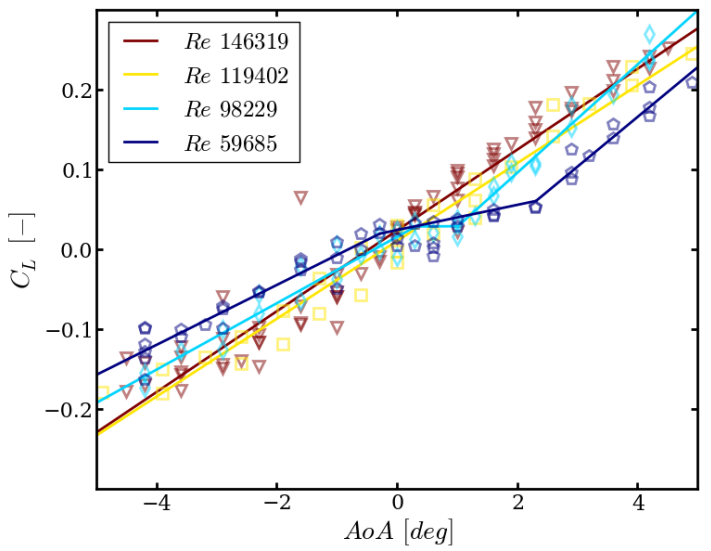

(a) Lift coefficient

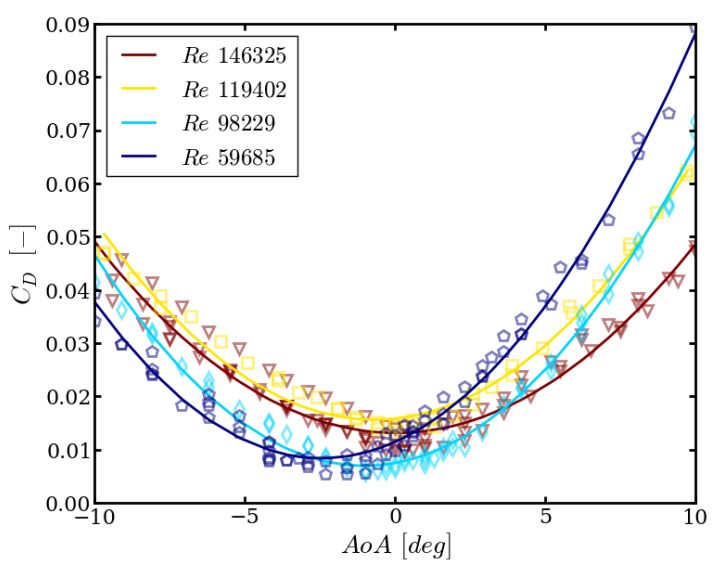

(b) Drag coefficient

Fig. 15. Measured lift and drag coefficients across a range of Reynolds numbers and angles of attack. Markers denote individual data points and solid lines represent least squares fits - piecewise linear for lift, quadratic for drag coefficients, respectively.

the shape of the foil would likely lead to an increase in drag rather than its reduction. A possible physical explanation of the asymmetry of the drag curve changing as a function of the Reynolds number may be that due to its shape varying between the upper and lower halves, each of them respond differently to the laminar separation, thus inducing an asymmetric drag response. As the transitional effects get reduced and ultimately vanish at higher Reynolds numbers, so does the asymmetry in the drag measurement.

\section{CONCLUDING REMARKS}

\section{A. Conclusions}

Successful lift and drag measurements have been conducted on a scaled version of an underwater glider appendage. The experimental set up developed has been tailored to suit the demanding needs of the tests carried out in terms of being able to capture the small magnitudes of forces induced by the foil. While the accuracy of the drag results could be improved, the overall trends observed in the data agree with similar measurements and simulations by other authors. This shows that while certainly challenging, direct measurements of low$\mathrm{Re}$ aerofoil forces are possible using low-cost experimental procedures.

The data clearly show that even a geometry which is not exceptionally smooth nor placed in a flow with very low turbidity exhibits laminar and transitional flow behaviours. These will have an important effect on performance of underwater vehicles operating at low Reynolds numbers, such as underwater gliders or small conventional AUVs. Accurately accounting for those effects is therefore important from the point of view of vehicle design, dynamic modelling and model-predictive control, as well as mission planning.

\section{B. Future work}

The next stages of the present on-going investigation will focus predominantly on classifying the performance of underwater vehicle rudders and other control surfaces, as well as several other hydrofoil designs. This information will then be used to improve the design of a new underwater glider, as well as provide validation data for numerical prediction methods.

While successful, the present experimental set up could benefit from several refinements and improvements. The addition of an integrated thermometer, an atmospheric pressure sensor, and a wind speed gauge connected to the data acquisition system, would allow detailed information on the variation of these properties over the duration each acquisition run to be obtained and factored into the analysis. Employing a digital inclinometer would help in reducing vertical alignment uncertainty. Furthermore, reducing the drag readings due to lift would reduce the dependence of the measurements on the correction terms, thus reducing uncertainty. Achieving this would require structural modifications to the force block or the use of more expensive sensors. Increasing the signal-to-noise ratio and sensitivity of the drag transducers would make the low-Re operation more reliable. This could be done by using different sensors or refining the amplifier circuits. Finally, the currently selected 3D printing strategy should be improved in order to ensure better symmetry of the manufactured foils.

\section{ACKNOWLEDGEMENTS}

The presented work has been funded as a part of the BRIDGES Project (http://www.bridges-h2020.eu/). This Project has received funding from the European Unions Horizon 2020 research and innovation programme under grant agreement No 635359.

\section{REFERENCES}

[1] D. L. Rudnick, R. E. Davis, C. C. Eriksen, D. M. Fratantoni, and M. J. Perry, "Underwater Gliders for Ocean Research," Marine Technology Society Journal, vol. 38, no. 2, pp. 73-84, 2004.

[2] J. Sherman, R. E. Davis, W. B. Owens, and J. Valdes, "The autonomous underwater glider," Oceanic Engineering, IEEE Journal of, vol. 26 , no. 4, pp. 437-446, 2001.

[3] A. K. Lidtke, S. R. Turnock, and J. Downes, "Assessment of Underwater Glider Performance Through Viscous Computational Fluid Dynamics," in Autonomous Underwater Vehicles 2016 (AUV), (6-9 November, IIS, the University of Tokyo, Tokyo, Japan), 2016. 
[4] S. Lemaire, A. K. Lidtke, G. Vaz, and S. R. Turnock, "Modelling Natural Transition on Hydrofoils for Application in Underwater Gliders," in 19th Numerical Towing Tank Symposium (NuTTS), (3-4 October, St Pierre d'Oleron, France), 2016

[5] L. Parras and C. Pino, "Comparison of the Aerodynamic Characteristics of the NACA0012 Airfoil at Low-to-Moderate Reynolds Numbers for any Aspect Ratio," International Journal of Aerospace Sciences, vol. 4, no. 1 , pp. 1-8, 2016

[6] G. K. Ananda, P. P. Sukumar, and M. S. Selig, "Low-to-Moderate Aspect Ratio Wings Tested at Low Reynolds Numbers," in 30th AIAA Applied Aerodynamics Conference, no. 25-28 June, (New Orleans, Loisiana, USA), pp. 1-19, 2012

[7] W. G. J. Bastedo and T. J. Muellert, "Spanwise Variation of Laminar Separation Bubbles on Wings at Low Reynolds Numbers," Journal of Aircraft, vol. 23, no. 9, 1986.

[8] M. Mizoguchi and H. Itoh, "Effect of Aspect Ratio on Aerodynamic Characteristics at Low," AIAA Journal, vol. 51, no. 7, 2013.

[9] G. E. Torres and T. J. Mueller, "Low-Aspect-Ratio Wing Aerodynamics at Low Reynolds Numbers," AIAA Journal, vol. 42, no. 5, 2004.

[10] S. C. Yen and L.-C. Huang, "Flow Patterns and Aerodynamic Performance of Unswept and Swept-Back Wings," Journal of fluids engineering, vol. 131, no. November, pp. 1-10, 2009.

[11] M. S. Selig, J. J. Guglielmo, A. P. Broeren, and P. Giguere, "Summary of Low-speed airfoil data, volume 1," tech. rep., SoarTech Publications, Virginia Beach, Virginia, 1995.

[12] A. F. Molland, "The design, construction and calibration of a fivecomponent strain gauge wind tunnel dynamometer, Ship Science Repor 1/77," tech. rep., University of Southampton, Southampton, UK, 1976.

[13] I. P. Castro, "Personal communication," 2017.

[14] A. F. Molland and S. R. Turnock, Marine rudders and control surfaces. London: Elsevier, 1 ed., 2007. 